\title{
A gustocentric perspective to understanding primary sensory cortices.
}

\author{
Roberto Vincis ${ }^{1}$ and Alfredo Fontanini ${ }^{1,2}$. \\ ${ }^{1}$ Department of Neurobiology and Behavior and ${ }^{2}$ Program in Neuroscience, State University of New York \\ at Stony Brook, Stony Brook, NY, 11794, USA.
}

Correspondence should be addressed to R.V. (roberto.vincis@stonybrook.edu) or to A.F. (alfredo.fontanini@stonybrook.edu).

Acknowledgments: The authors would like to thank Drs. Arianna Maffei, Giancarlo La Camera and Luca Mazzucato for helpful discussions and insightful feedback. R.V. was funded by Swiss National Science Foundation Fellowships P2GEP3_151816 and P300PA 161021; A.F. was funded by National Institute of Deafness and Other Communication Disorders Grant R01-DC012543.

(C) 2016. This manuscript version is made available under the Elsevier user license http://www.elsevier.com/open-access/userlicense/1.0/ 


\begin{abstract}
Most of the general principles used to explain sensory cortical function have been inferred from experiments performed on neocortical, primary sensory areas. Attempts to apply a neocortical view to the study of the gustatory cortex (GC) have provided only a limited understanding of this area. Failures to conform GC to classical neocortical principles have been implicitly interpreted as a demonstration of $\mathrm{GC}^{\prime}$ s uniqueness. Here we propose to take the opposite perspective, dismissing $\mathrm{GC}^{\prime} \mathrm{s}$ uniqueness and using principles extracted from its study as a lens for looking at neocortical sensory function. In this review, we describe three significant findings related to gustatory cortical function and advocate their relevance for understanding neocortical sensory areas.
\end{abstract}




\section{Introduction}

Historically, sensory physiologists interested in understanding the computations performed by cortical circuits have focused their attention on neocortical areas [1-3]. The ability to precisely control the physical variables of a stimulus, as well as the ease of experimental access have contributed to rendering visual, somatosensory and auditory cortices primary models for investigating sensory processing. Electrophysiological studies from these areas started earlier and engaged larger communities than studies on chemosensory cortices. As a result, many of the fundamental principles of sensory, cortical physiology have been defined by results obtained in these cortices. The cortical organization in sensory maps $[2,4,5]$, the presence of columns [1] and stereotyped circuits [6] and the hierarchical organization of sensory streams (i.e. "lower" order areas devoted to signal processing and "higher" order areas involved in integration) [7] are just few among the many principles established in neocortical areas. Albeit never explicitly theorized, the knowledge accumulated on neocortical areas has influenced the way in which chemosensory areas have been approached. Multiple attempts have been made to adapt general principles of neocortical processing to either olfactory or gustatory cortices [8-10]. Failures to fit some of such principles to chemosensory areas have been interpreted as evidence for the uniqueness of these cortices. However, many of the results initially deemed as specific to chemosensory cortices have proven to generalize to neocortical areas. For instance, studies of oscillations and their links to sensory coding and cognition have been pioneered in the olfactory system [11-14]. Similarly, the importance of sensorimotor rhythms has been first established by looking at sniffing and respiration [15-18]. The recent rise in attention toward the olfactory cortex has brought into focus its relevance for understanding general computational principles. However, a similar process has not yet occurred for the gustatory cortex (GC). In the case of GC, the results have been either viewed as confirmatory of known

neocortical principles $[8,9]$, or, if unique and unprecedented, treated as an exotic peculiarity. In this review, we will discuss recent developments in understanding the function of the gustatory cortex, with the goal of showing how many of the findings on this area can help us gain an original perspective on neocortical sensory areas.

\section{Coding of chemosensory information: the importance of time and dynamics.}

Neurons in the gustatory cortex are responsible for mediating the perception of different taste qualities: sweet, salty, sour, bitter and umami (just to name the best studied). GC neurons encode chemosensory information via time-varying changes in firing rates $[19,20]$. Initial analyses of the time course of firing rate activity revealed a great richness in single neuron responses to intraorally delivered tastants [20]. 
Most of the firing rate modulations revolved around three temporal epochs that encode different aspects of sensory information and unfold on the relatively long-lasting period of $2.5 \mathrm{~s}$ from taste delivery [20] [19,21-23].

The temporal evolution of taste-evoked activity in GC has also been analyzed at the neural ensemble level. Use of the Hidden Markov Model (HMM) to extract specific patterns of ensemble activity revealed that upon gustatory stimulation populations of neurons in GC go through different states of partially coordinated activity [24-28]. Each state can last from few to hundreds of milliseconds and suddenly end, leading the network to a rapid transition into another state. Multiple lines of evidence prove the importance of this regime of activity. Analysis of responses to multiple tastants revealed that each taste quality is associated with a specific sequence of states [26]. While the duration of each state and the times at which transitions occur vary from trial-to-trial, each particular sequence of states is specific to a certain taste. The sequences generated by a HMM analysis allowed for a very effective decoding of stimulus identity, showing that this conceptual and analytical framework successfully captures how GC encodes sensory information $[25,26]$. This approach to understanding how GC encodes gustatory information goes beyond the traditional single cell perspective, based on tuning curves computed from averaged data (Figure 1A), and suggests that sensory cortical processing entails the concerted activity of populations of neurons.

Understanding sensory cortical activity in terms of metastable states allowed for insights beyond sensory coding. The description of sudden transitions between states lead to the suggestion that a stochastic, jumping model of decision making could account for ingestive decisions more accurately than the traditional diffusion-to-bound model, particularly in conditions of noisy signals [29,30] (Figure 1). Recent data confirmed this suggestion in both GC [31] and lateral intraparietal area (LIP) [32]. Finally, recent work unveiled that metastability is not limited to evoked activity, but can also be observed during spontaneously ongoing activity [28] (Figure 1d). Populations of neurons in GC undergo sudden jumps between states even in the absence of any overt stimulation. Analysis of firing rates of single neurons revealed that metastability is not simply caused by neurons alternating between two regimes of firing rates. Rather, neurons can produce multiple (i.e. more than two) firing rates depending on the state (a feature called multistability). Spontaneous metastability and multistability result from network interactions, as demonstrated by theoretical analysis and simulations with a network model of spiking neurons [28]. Such a model features balanced excitation/inhibition and a clustered architecture and generates an attractor landscape that spontaneous activity explores (Figure 1e). The model built to explain spontaneous activity could reproduce fundamental features of stimulus-evoked activity $[27,28]$, 
indicating that the elusive relationship between spontaneous and evoked cortical activity [33] is grounded in the dynamics internally generated by a network with clustered architecture [34].

GC represents the first primary cortical sensory area for which the study of metastable ensemble dynamics provides a unifying view that explains sensory coding, decision-making and the relationship between evoked and spontaneous activity. We believe that this approach could also be productively adopted to study other primary sensory areas, beyond taste and olfaction [35].

\section{Integration between sensory and reward processing.}

Taste coding is intimately linked with reward processing. Gustatory stimuli have hedonic valence, they are either palatable or aversive. The reward value of taste can be easily measured relying either on consummatory behaviors $[36,37]$ or orofacial reactions [38,39]. The ability to assess objectively different dimensions of reward with specific behavioral tests has allowed researchers in the field to explore the involvement of GC in processing reward. Spatio-temporal patterns of neural activity in GC account for the palatability and/or aversiveness of taste. Imaging experiments in rodents revealed that the spatial representations of taste qualities are plastic and can track changes in the valence of gustatory stimuli [40]. Extensive analysis of single neuron responses showed that valence is encoded by the temporal structure of firing rate changes $[21,23,41]$. Specifically, it has been demonstrated that valence coding emerges with a slow latency (i.e., 1 second) compared to stimulus onset. Population analyses with HMM confirmed this observation and provided further evidence indicating that, at a single trial level, valence coding emerges as a sudden and rapid change in the state of ensemble activity [31]. Recent ensemble recordings in rat GC showed that gapes (i.e. orofacial reactions of aversion) were reliably preceded by a state change, suggesting a causal role of GC in evaluating the palatability of gustatory stimuli. Results from optogenetic studies confirm GC's involvement in mediating appetitive and aversive responses [42]. The sources of valence-related information in GC could be multiple, given its connectivity with limbic regions [43]. However, clear evidence points at the amygdala as one of the main synaptic inputs to GC $[44,45]$ and a fundamental source of reward-related signals $[41,46,47]$.

The intimate relationship between taste and reward implies that gustatory stimuli can also be used as outcomes. Using tastants as unconditioned stimuli in learning paradigms led to the discovery that GC plays a role in encoding reward-predictive stimuli $[46,48-50]$. Training rats to associate a predictive cue with the availability of multiple tastants unveiled that GC neurons can be modulated by non-gustatory, anticipatory cues. The appearance of cue responses, a phenomenon observed so far only in limbic areas depends on learning, and can be observed with both classical and instrumental conditioning paradigms 
$[46,48,49]$. In addition, neurons in GC can encode both the general expectation of taste, i.e. the expectation that a general gustatory stimulus is going to be available regardless of its identity, and the specific expectation of tastants with opposite valence [49] (Figure 2). The obvious question, at this point, is whether this cue-related activity plays any role in guiding behavior, or is just a byproduct of GC's connectivity with limbic areas. Behavioral studies relying on pharmacological manipulations of the activity in GC suggested its involvement in guiding behavioral choices [51]. A recent study was specifically designed to investigate the role of cue-related activity in GC [52]. Mice were trained to associate the delivery of a food pellet at a food-port with a 10 seconds long anticipatory cue. Upon successful training, mice responded to the cue with a series of conditioned, food-port entries. Inactivation of GC selectively during the presentation of the cue significantly reduced the number of food-port entries, indicating that cue-related activity in GC affects behavior.

While one might argue that taste is unique among the senses for its intimate relationship with reward, this is certainly not the case. Stimuli of all sensory modalities can have an affective dimension, either innately or upon experience. Alas, only a few studies have focused on such an important dimension of perception in primary sensory areas other than GC [53-57].

\section{Multisensoriality in a primary sensory cortex.}

Neurons in GC are not devoted exclusively to processing the physiochemical and affective dimensions of taste. Multiple studies demonstrate that GC can effectively process non-gustatory, cross-modal stimuli encountered either during the consumption of food or even before [19,46,49,58-62].

Evidence for multisensory integration during consumption comes from experiments showing that single neurons in GC can encode tactile, thermal and olfactory information coming from the oral cavity $[61,63]$. The integration of these multimodal, intraoral signals relates to the ability of perceiving flavor [64-66], the unitary, multisensory percept associated with food consumption. A second form of multisensory processing, fundamental for forming expectations, can occur in GC prior to the consumption of food and triggered by extraoral stimuli $[46,49]$. The sight of food, for instance, successfully activates GC in human subjects [67]. In alert rats, GC neurons can respond to sounds, or odors, predicting either the general availability of taste or specific gustatory stimuli $[46,49]$ (Figure 2). Single unit recordings and immediate early gene analysis demonstrated that cross-modal responses are present before learning and increase in prevalence and specificity after stimulus-taste associations $[46,49,50]$.

While one might argue that the multimodality of GC is a direct consequence of the intrinsic relationship of tastants with tactile and odor stimuli, or a consequence of taste being always preceded by other 
sensory stimuli, this is not the case. In the real world, each unimodal sensory stimulus is perceived against the background of other stimuli. Perception is inherently associated with multisensory integration. While few studies have indeed investigated cross-modal responses in primary sensory areas $[56,57,68]$, we believe not enough attention has been devoted to this fundamental issue.

\section{Conclusion}

In this brief overview, we discussed three lines of investigation on the function of GC. These approaches have not been directly influenced by existing theories of neocortical sensory function. Rather, these were efforts directed at understanding $\mathrm{GC}^{\prime}$ s relationship to gustatory perception and taste-related behaviors. These research directions have contributed to 1 ) demonstrate that a single computational framework can link sensory coding, sensory-based decisions and spontaneous activity; 2) unveil the intimate links between perception and reward; 3) define sensory processing in primary cortices as an inherently multisensory process. Altogether, this research has shown that the primary gustatory cortex is not simply an analyzer of physiochemical information, but rather an area that integrates perception, emotion and actions. Of course, one could dismiss all the results from GC as peculiarities of a "unique" cortex. After all, one might argue, GC is part of the insula (an area known to be integrative), has a unique cytoarchitecture and deals with atypical stimuli. Here we contend that the opposite attitude may be more productive and could lead to original insights on the function of neocortical sensory areas. Time might have come to stop imposing a neocortical view on all the sensory cortices and begin to be inspired by the chemical senses.

\section{Legends}

Figure 1: Metastable dynamics in the gustatory cortex of awake animals. a) Representative ensemble showing taste-evoked metastable dynamics. Top: population raster with overlaid the HMM fit; each color represents a different state. Bottom: histograms showing firing rate vectors for each HMM state. b) Metastable dynamics and taste quality coding. Left: schematic showing the four different tastants delivered. Right: histogram showing that an HMM-based classification can outperform a PSTH-based classification. c) Metastable dynamics and decision-making. Left: schematic showing the architecture of the decision-making network used to test the role of reproduce metastable in ingestive decisions. Right. Comparison of the performance for two models of decision-making: deterministic integration ("ramping") and stochastic ("jumping"). The plot shows how performance depends on network size and internal noise. For a complete analysis of the differences between the two models of decision-making 
see Miller and Katz [30] d) Representative example of metastable dynamics during spontaneous activity. Top: population raster with overlaid the HMM fit; each color represents a different state. Bottom: histograms showing firing rate vectors for each state. e) Model and theory of spontaneous activity. Left: schematic of the clustered spiking network capable of reproducing spontaneous metastability and multistability. Right: Attractor landscape of the spiking network model. The plot shows the number of active clusters and relative firing rates of neurons inside clusters as a function of the strength of relative intracluster synaptic connections $\left(J_{+}\right)$. Vertical dotted lines indicate values of intracluster connections at which new clusters are recruited. The numbers of activated clusters in each configuration are indicated in red (configurations with larger clusters have lower firing rates, due to the overall inhibition). Panel a) and b) modified from Jones et al [26]; panel c) modified from Miller and Katz [29]; panel e) modified from Mazzucato et al [28].

Figure 2: Cross-modal cue responses in the gustatory cortex can encode specific expectations of rewarding or aversive tastants. a) Go/no go paradigm. Top: schematic of the behavioral paradigm; one auditory cue predicts the availability of sucrose; the other cue predicts the availability of quinine. Rats press a lever following the sucrose predicting cue (Suc_Cue) and self-deliver sucrose directly into their mouth. On the contrary, rats refrain from pressing following the quinine-predicting cue (Q_Cue). Bottom: representative behavioral record a session. The plot shows the percentage of trials for Suc_Cue (cyan) and Q_Cue (gold) followed by a lever pressing. The solid black line indicates the average performance. b) Selectivity of cue responses. Raster plots and peri-stimulus histograms (PSTHs) for two representative units, one selective for Suc_Cue (blue, left) and one selective for Q_Cue (right, gold). The dotter line overlaying the PSTHs shows the time course of the magnitude of mouth movements. Time 0 represents the onset of the auditory cue. Triangles markers represent lever-presses. The gray shading indicates the temporal window in which only the cue was present, without any lever-press. c) Emergence of cue selectivity with learning. The three plots depict the difference in normalized firing between responses to Suc_Cue and Q_Cue averaged across neurons. A flat line indicates no difference between cue responses (hence no cue selectivity). Each plot features data from animals at different stage of learning. Top: rats not showing any sign of learning in the first few sessions; middle: rats showing learning in the first few sessions; bottom: rats extensively trained. Notice how the difference between cue responses (i.e., the selectivity) increases with learning. Time 0 and gray shadow as in panel 
b. Dotted horizontal line represents zero difference. Panels a-c) modified from Gardner and Fontanini [49]. 


\section{References}

1. Mountcastle VB: The columnar organization of the neocortex. Brain 1997, 120 ( Pt 4):701-722.

2. Hubel DH, Wiesel TN: Ferrier lecture. Functional architecture of macaque monkey visual cortex. Proc $R$ Soc Lond B Biol Sci 1977, 198:1-59.

3. Brugge JF, Merzenich MM: Responses of neurons in auditory cortex of the macaque monkey to monaural and binaural stimulation. J Neurophysiol 1973, 36:1138-1158.

4. Kaas JH: The functional organization of somatosensory cortex in primates. Ann Anat 1993, 175:509518.

5. Reale RA, Imig TJ: Tonotopic organization in auditory cortex of the cat. J Comp Neurol 1980, 192:265291.

6. Douglas RJ, Martin KA: Neuronal circuits of the neocortex. Annu Rev Neurosci 2004, 27:419-451.

7. Felleman DJ, Essen DC: Distributed hierarchical processing in the primate cerebral cortex. Cerebral cortex 1991, 1:1-47.

8. Sato H, Shimanuki Y, Saito M, Toyoda H, Nokubi T, Maeda Y, Yamamoto T, Kang Y: Differential columnar processing in local circuits of barrel and insular cortices. The Journal of neuroscience : the official journal of the Society for Neuroscience 2008, 28:3076-3089.

9. Chen X, Gabitto M, Peng Y, Ryba NJ, Zuker CS: A gustotopic map of taste qualities in the mammalian brain. Science 2011, 333:1262-1266.

10. Sewards TV, Sewards MA: Cortical association areas in the gustatory system. Neurosci Biobehav Rev 2001, 25:395-407.

11. Adrian ED: Olfactory reactions in the brain of the hedgehog. J Physiol 1942, 100:459-473.

12. Wilson $\mathrm{M}$, Bower JM: Cortical oscillations and temporal interactions in a computer simulation of piriform cortex. J Neurophysiol 1992, 67:981-995.

13. Eeckman FH, Freeman WJ: Correlations between unit firing and EEG in the rat olfactory system. Brain research 1990, 528:238-244.

14. Kay LM, Beshel J, Brea J, Martin C: Olfactory oscillations: the what, how and what for. Trends in ... 2009.

15. Fontanini A, Bower JM: Slow-waves in the olfactory system: an olfactory perspective on cortical rhythms. Trends Neurosci 2006, 29:429-437.

16. Macrides F, Eichenbaum HB, Forbes WB: Temporal relationship between sniffing and the limbic theta rhythm during odor discrimination reversal learning. J Neurosci 1982, 2:1705-1717.

17. Verhagen JV, Wesson DW, Netoff TI, White JA, Wachowiak M: Sniffing controls an adaptive filter of sensory input to the olfactory bulb. Nat Neurosci 2007, 10:631-639.

18. Wesson DW, Donahou TN, Johnson MO, Wachowiak M: Sniffing behavior of mice during performance in odor-guided tasks. Chem Senses 2008, 33:581-596.

19. Katz DB, Simon SA, Nicolelis MA: Dynamic and multimodal responses of gustatory cortical neurons in awake rats. $J$ Neurosci 2001, 21:4478-4489.

20. Katz DB, Nicolelis MA, Simon SA: Gustatory processing is dynamic and distributed. Current opinion in neurobiology 2002, 12:448-454.

21.* Sadacca BF, Rothwax JT, Katz DB: Sodium concentration coding gives way to evaluative coding in cortex and amygdala. J Neurosci 2012, 32:9999-10011.

This study relies on multielectrode recordings in the gustatory cortex and amygdala of alert rats receiving increasing concentrations of $\mathrm{NaCl}$. The authors show how these two areas multiplex information related to stimulus intensity and stimulus palatability. 
22. Jezzini A, Mazzucato L, La Camera G, Fontanini A: Processing of hedonic and chemosensory features of taste in medial prefrontal and insular networks. J Neurosci 2013, 33:18966-18978.

23. Grossman SE, Fontanini A, Wieskopf JS, Katz DB: Learning-related plasticity of temporal coding in simultaneously recorded amygdala-cortical ensembles. J Neurosci 2008, 28:2864-2873.

24. Moran A, Katz DB: Sensory cortical population dynamics uniquely track behavior across learning and extinction. $J$ Neurosci 2014, 34:1248-1257.

25. Escola S, Fontanini A, Katz D, Paninski L: Hidden Markov models for the stimulus-response relationships of multistate neural systems. Neural Comput 2011, 23:1071-1132.

26. Jones LM, Fontanini A, Sadacca BF, Miller P, Katz DB: Natural stimuli evoke dynamic sequences of states in sensory cortical ensembles. Proc Natl Acad Sci U S A 2007, 104:18772-18777.

27. Mazzucato L, Fontanini A, La Camera G: Stimuli Reduce the Dimensionality of Cortical Activity. Front Syst Neurosci 2016, 10:11.

28. **Mazzucato L, Fontanini A, La Camera G: Dynamics of multistable states during ongoing and evoked cortical activity. J Neurosci 2015, 35:8214-8231.

This article combines data analysis, spiking network modeling, and mean field theory to demonstrate the existence of metastable states during spontaneous activity in the gustatory cortex. In addition, a mechanistic explanation for the genesis of this activity is proposed.

29. **Miller P, Katz DB: Stochastic transitions between neural states in taste processing and decisionmaking. The Journal of neuroscience : the official journal of the Society for Neuroscience 2010, 30:2559-2570.

This modeling study proposes, for the first time, the importance of sudden changes in cortical activity in decision making.

30. Miller P, Katz DB: Accuracy and response-time distributions for decision-making: linear perfect integrators versus nonlinear attractor-based neural circuits. J Comput Neurosci 2013, 35:261294.

31. *Sadacca BF, Mukherjee N, Vladusich T, Li JX, Katz DB, Miller P: The Behavioral Relevance of Cortical Neural Ensemble Responses Emerges Suddenly. J Neurosci 2016, 36:655-669.

This article demonstrates that aversive reactions to taste are reliably preceded by a sudden change in GC ensemble activity.

32. Latimer KW, Yates JL, Meister ML, Huk AC, Pillow JW: NEURONAL MODELING. Single-trial spike trains in parietal cortex reveal discrete steps during decision-making. Science 2015, 349:184187.

33. Arieli A, Sterkin A, Grinvald A, Aertsen A: Dynamics of ongoing activity: explanation of the large variability in evoked cortical responses. Science 1996, 273:1868-1871.

34. Kiani R, Cueva CJ, Reppas JB, Peixoto D, Ryu SI, Newsome WT: Natural grouping of neural responses reveals spatially segregated clusters in prearcuate cortex. Neuron 2015, 85:1359-1373.

35. Laurent G, Stopfer M, Friedrich RW, Rabinovich MI, Volkovskii A, Abarbanel HD: Odor encoding as an active, dynamical process: experiments, computation, and theory. Annual review of neuroscience 2001, 24:263-297.

36. Davis JD, Smith GP: Analysis of the microstructure of the rhythmic tongue movements of rats ingesting maltose and sucrose solutions. Behav Neurosci 1992, 106:217-228.

37. Schier LA, Spector AC: Behavioral Evidence for More than One Taste Signaling Pathway for Sugars in Rats. The Journal of neuroscience : the official journal of the Society for Neuroscience 2016, 36:113-124. 
38. Delamater AR, LoLordo VM, Berridge KC: Control of fluid palatability by exteroceptive Pavlovian signals. J Exp Psychol Anim Behav Process 1986, 12:143-152.

39. Grill HJ, Norgren R: The taste reactivity test. I. Mimetic responses to gustatory stimuli in neurologically normal rats. Brain Res 1978, 143:263-279.

40. Accolla $R$, Carleton $A$ : Internal body state influences topographical plasticity of sensory representations in the rat gustatory cortex. Proc Natl Acad Sci U S A 2008, 105:4010-4015.

41. Piette CE, Baez-Santiago MA, Reid EE, Katz DB, Moran A: Inactivation of basolateral amygdala specifically eliminates palatability-related information in cortical sensory responses. $J$ Neurosci 2012, 32:9981-9991.

42. Peng Y, Gillis-Smith S, Jin H, Trankner D, Ryba NJ, Zuker CS: Sweet and bitter taste in the brain of awake behaving animals. Nature 2015, 527:512-515.

43. Maffei A, Haley M, Fontanini A: Neural processing of gustatory information in insular circuits. Curr Opin Neurobiol 2012, 22:709-716.

44. Stone ME, Maffei A, Fontanini A: Amygdala stimulation evokes time-varying synaptic responses in the gustatory cortex of anesthetized rats. Front Integr Neurosci 2011, 5:3.

45. Haley MS, Fontanini A, Maffei A: Laminar- and Target-Specific Amygdalar Inputs in Rat Primary Gustatory Cortex. The Journal of neuroscience : the official journal of the Society for Neuroscience 2016, 36:2623-2637.

46. Samuelsen CL, Gardner MP, Fontanini A: Effects of cue-triggered expectation on cortical processing of taste. Neuron 2012, 74:410-422.

47. Bermudez-Rattoni $F$ : The forgotten insular cortex: its role on recognition memory formation. Neurobiol Learn Mem 2014, 109:207-216.

48. Samuelsen CL, Gardner MP, Fontanini A: Thalamic contribution to cortical processing of taste and expectation. J Neurosci 2013, 33:1815-1827.

49. **Gardner MP, Fontanini A: Encoding and tracking of outcome-specific expectancy in the gustatory cortex of alert rats. J Neurosci 2014, 34:13000-13017.

The results of this article demonstrate that neurons in the gustatory cortex can selectively encode auditory cues predicting different tastants. Selectivity of cue responses develops with learning, tracks the anticipatory value of the cue and predicts responses to taste.

50. Saddoris MP, Holland PC, Gallagher M: Associatively learned representations of taste outcomes activate taste-encoding neural ensembles in gustatory cortex. J Neurosci 2009, 29:1538615396.

51. Parkes SL, Balleine BW: Incentive memory: evidence the basolateral amygdala encodes and the insular cortex retrieves outcome values to guide choice between goal-directed actions. J Neurosci 2013, 33:8753-8763.

52. Kusumoto-Yoshida I, Liu H, Chen BT, Fontanini A, Bonci A: Central role for the insular cortex in mediating conditioned responses to anticipatory cues. Proc Natl Acad Sci U S A 2015, 112:11901195.

53. Namboodiri VM, Huertas MA, Monk KJ, Shouval HZ, Hussain Shuler MG: Visually cued action timing in the primary visual cortex. Neuron 2015, 86:319-330.

In this study the authors present compelling evidence that neurons in the visual cortex are involved in the decision to time visually-guided actions. The results are based on electrophysiological recordings, optogenetics and modeling.

54. Pantoja J, Ribeiro S, Wiest M, Soares E, Gervasoni D, Lemos NA, Nicolelis MA: Neuronal activity in the primary somatosensory thalamocortical loop is modulated by reward contingency during 
tactile discrimination. The Journal of neuroscience : the official journal of the Society for Neuroscience 2007, 27:10608-10620.

55. Gire DH, Whitesell JD, Doucette W, Restrepo D: Information for decision-making and stimulus identification is multiplexed in sensory cortex. Nature neuroscience 2013, 16:991-993.

56. Brosch M, Selezneva E, Scheich $\mathrm{H}$ : Nonauditory events of a behavioral procedure activate auditory cortex of highly trained monkeys. The Journal of neuroscience : the official journal of the Society for Neuroscience 2005, 25:6797-6806.

57. Zhou YD, Fuster JM: Visuo-tactile cross-modal associations in cortical somatosensory cells. Proc Natl Acad Sci U S A 2000, 97:9777-9782.

58. de Araujo IE, Simon SA: The gustatory cortex and multisensory integration. Int J Obes (Lond) 2009, 33 Suppl 2:S34-43.

59. Simon SA, de Araujo IE, Gutierrez R, Nicolelis MA: The neural mechanisms of gustation: a distributed processing code. Nat Rev Neurosci 2006, 7:890-901.

60. Rodgers KM, Benison AM, Klein A, Barth DS: Auditory, somatosensory, and multisensory insular cortex in the rat. Cereb Cortex 2008, 18:2941-2951.

61. Yamamoto T, Yuyama N, Kawamura Y: Cortical neurons responding to tactile, thermal and taste stimulations of the rat's tongue. Brain Res 1981, 221:202-206.

62. Yamamoto $T$, Matsuo R, Kiyomitsu $Y$, Kitamura R: Taste responses of cortical neurons in freely ingesting rats. J Neurophysiol 1989, 61:1244-1258.

63. Stapleton JR, Lavine ML, Wolpert RL, Nicolelis MA, Simon SA: Rapid taste responses in the gustatory cortex during licking. J Neurosci 2006, 26:4126-4138.

64. Spence C: Multisensory flavor perception. Cell 2015, 161:24-35.

65. Verhagen JV: The neurocognitive bases of human multimodal food perception: consciousness. Brain Res Rev 2007, 53:271-286.

66. Carleton A, Accolla R, Simon SA: Coding in the mammalian gustatory system. Trends Neurosci 2010, 33:326-334.

67. Ashkenazi A, Marks LE: Effect of endogenous attention on detection of weak gustatory and olfactory flavors. Percept Psychophys 2004, 66:596-608.

68. Iurilli G, Ghezzi D, Olcese U, Lassi G, Nazzaro C, Tonini R, Tucci V, Benfenati F, Medini P: Sounddriven synaptic inhibition in primary visual cortex. Neuron 2012, 73:814-828. 
(a)

Evoked activity

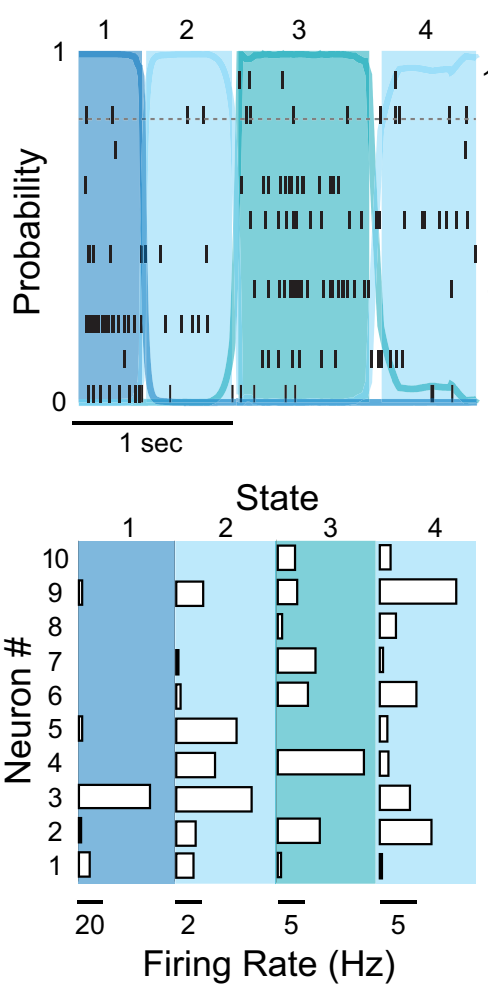

(d)

Spontaenous activity
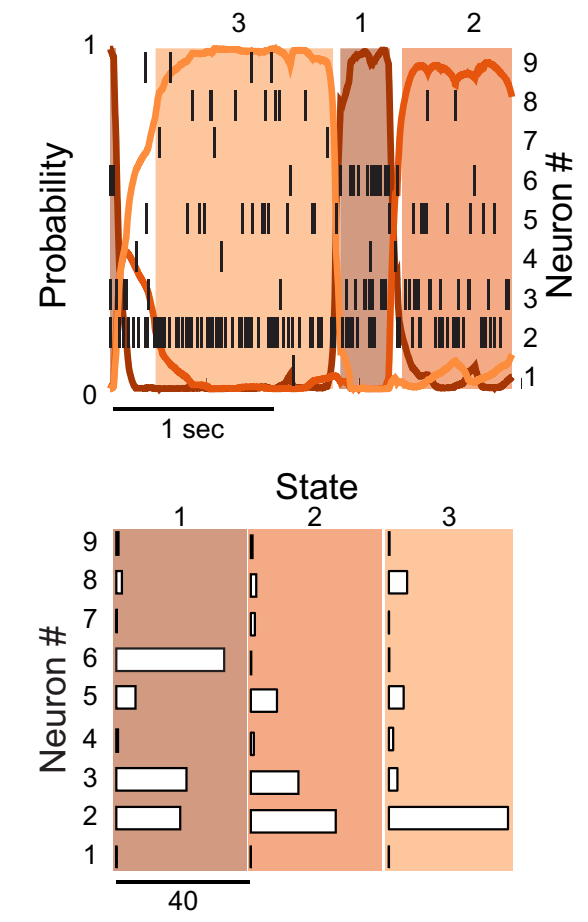

Firing Rate $(\mathrm{Hz})$ (b)
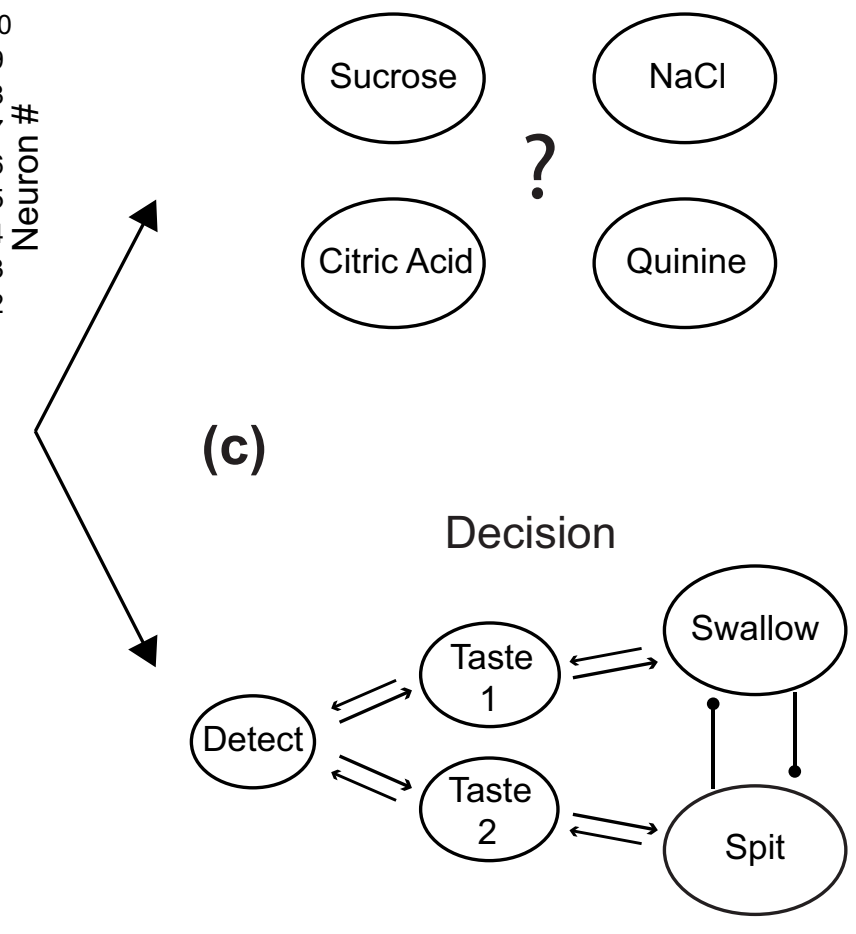

(e)

Model architecture

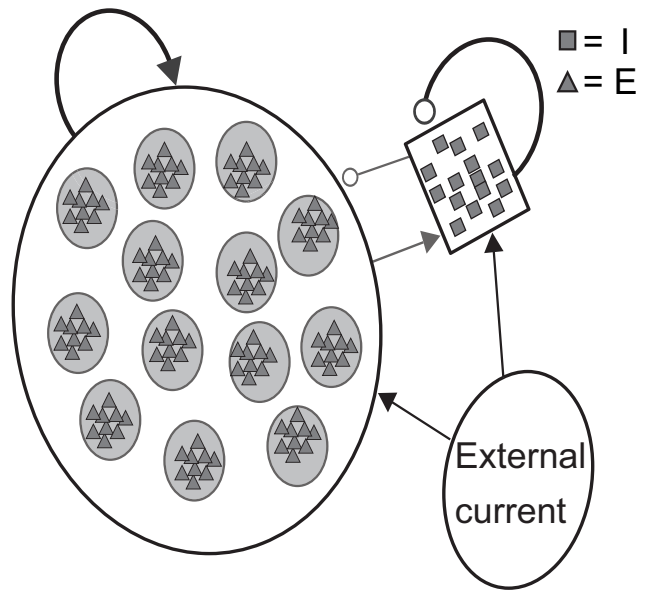

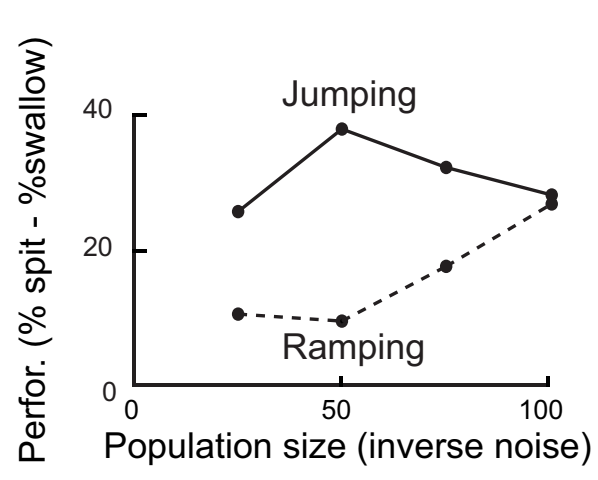

Stable states
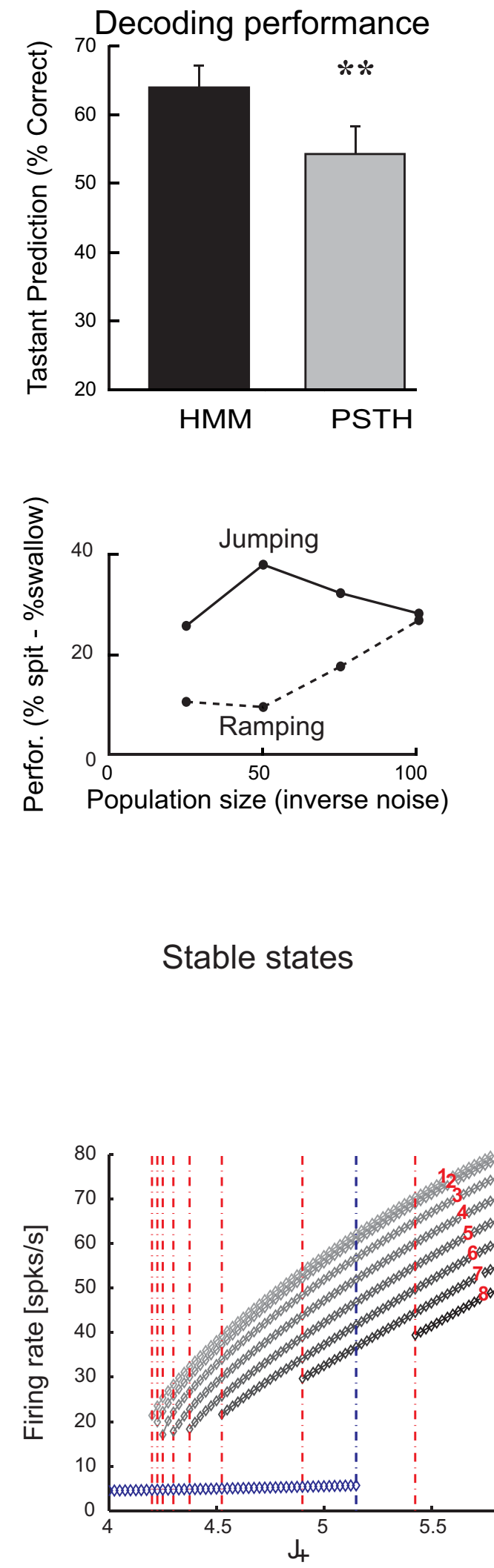
Figure_2

(a) Two Tone Go/No-Go Paradigm
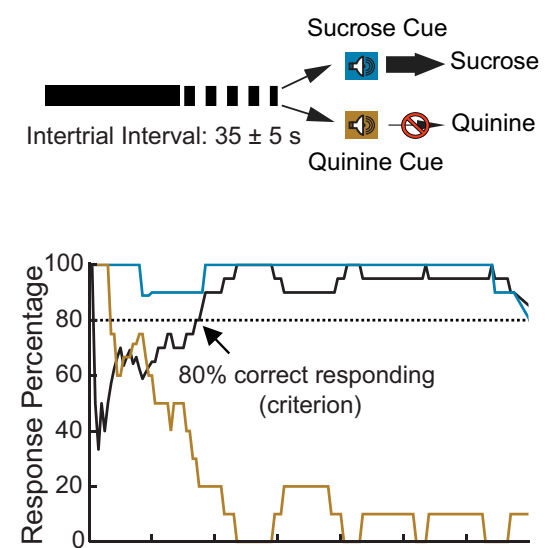

(1)

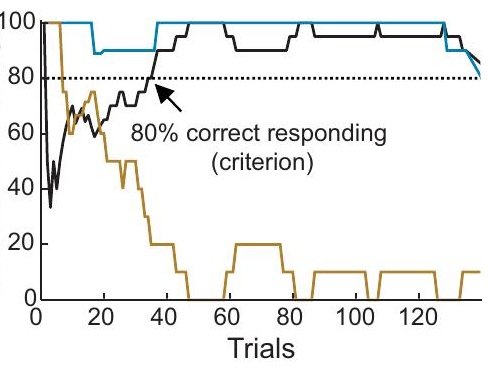

(b)

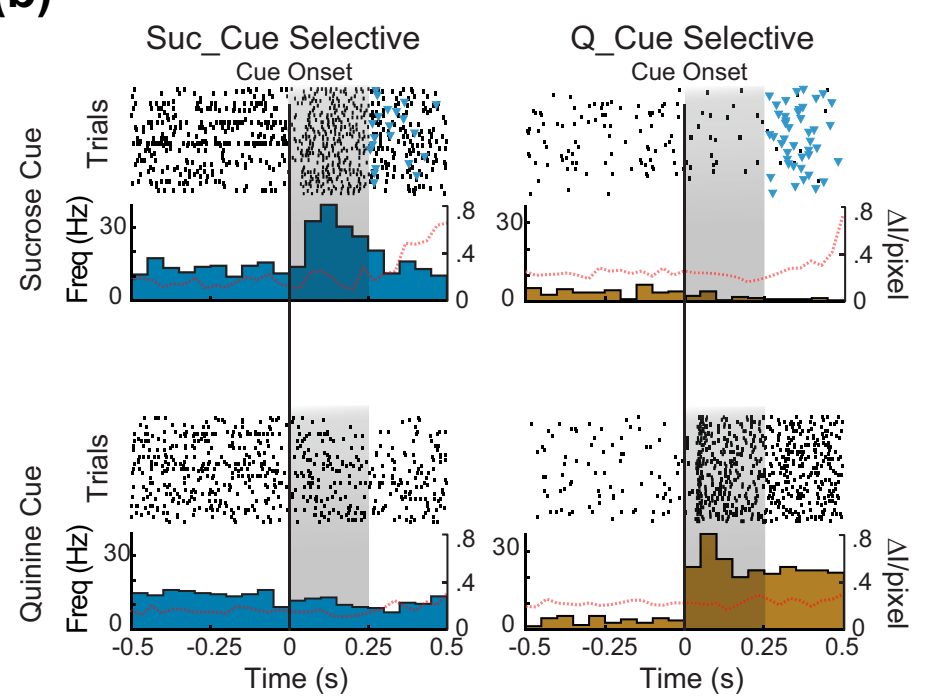

(c)

Difference between Suc_Cue and Q_Cue responses

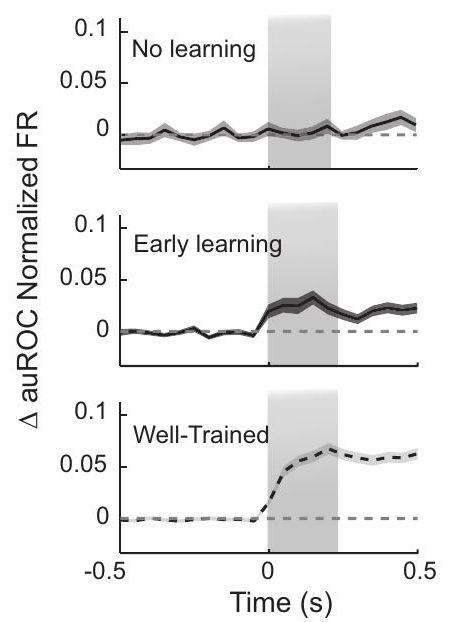

